\title{
HEMODYNAMIC CHANGES ASSOCIATED WITH SPINAL ANESTHESIA FOR CESAREAN DELIVERY IN SEVERE PREECLAMPSIA
}

\author{
By
Mahmoud El-Sayed Rashad Turkey, Abd El-Nasser Ahmed Hussein and Ali Abdallah Al-Kumity \\ Anesthesiology and Intensive Care Department, Faculty of Medicine, Al-Azhar University \\ * Corresponding author: Mahmoud El Sayed Rashad, \\ E-mail: turkeymahmoud808@gmail.com
}

\begin{abstract}
Background: Sever pre-eclampsia is defined as a disorder that occurs in pregnancy which manifests as hypertension and proteinuria with at least one maternal organ dysfunction involvement.

Objectives: To determine how pre-eclampsia affects maternal hemodynamics during cesarean delivery after spinal anesthesia.

Patients and Methods: This prospective randomized study was carried out on 30 normotensive females and 30 pre-eclamptic females admitted to Hospital for elective cesarean section using spinal anesthesia. The study was conducted in the Obstetrics Department of Al-Azhar University Hospitals, from January 2019 till December 2019.

Results: Incidence of hypotension after spinal anesthesia in group A (normotensive) was $66.7 \%$ and in group $\mathrm{B}$ (preeclampsia) was 30\%. The total dose of Ephedrine required to correct hypotension when it happen mean dose \pm SD in group A was $9.83 \pm 9.30$ and in group B was $3.00 \pm 5.15$. Neonatal outcomes (APGAR score and umbilical artery blood gases) were comparable. There was increased incidence of headache and blurring of vision among severe preeclampsia parturient.

Conclusion: Incidence of hypotension after spinal anaesthesiawas less frequent and less severe in preeclampsia patients and required lower dose of ephedrine than normotensive population. There was increased incidence of headache and blurring of vision among severe preeclampsia parturient. No difference in neonatal outcome between normotensive and severe preeclampsia population following spinal anesthesia.
\end{abstract}

Keywords: Hemodynamic, Spinal Anesthesia, Cesarean Delivery, Severe Preeclampsia.

\section{INTRODUCTION}

Features of severe preeclampsia include: Blood pressure > 159/109 mm Hg on two occasions at least 6 hrs. Apart, proteinuria of $\geq 5 \mathrm{~g}$ protein in 24-hr urine specimen or dipstick reading of $\geq 3+$ on two samples taken at least $4 \mathrm{hrs}$. apart, oliguria ( $<500 \mathrm{~mL}$ in $24 \mathrm{hrs}$.), pulmonary edema or cyanosis, abnormal liver function, right upper quadrant or epigastric pain, cerebral disturbances and thrombocytopenia (Tranquilli et al.,2013). In women with severe disease, the goal is detection of the development of organ dysfunction.

Pre-eclampsia remains an important cause of hypertensive acute pulmonary edema in pregnancy. There are a number of commonly associated clinical symptoms (breathlessness, orthopnea, 
agitation, and cough) and signs (tachycardia, tachypnea, crackles and wheeze on chest auscultation, cardiac S3 gallop, and decreased oxygen saturation) (Taydeet al., 2018).

The use of spinal anesthesia in preeclamptic pregnant woman is of considerable benefit, as these patients present particular hazards with general anesthesia, such as concerns for rapid airway control and cerebral blood flow alterations during induction of general anesthesia and intubation (Turneret al., 2010). However, the incidence of hypotension is high during spinal anesthesia for CS and it may approach values up to $95 \%$ (Banerjeeet al., 2010).

Hypotension during spinal anesthesia for cesarean delivery is a result of decreased vascular resistance due to sympathetic blockade and decreased cardiac output due to blood pooling in blocked areas of the body (Liguoriet al., 2012). Hypotension occurring during Cesarean section or following regional anesthesia is treated by careful fluid therapy and vasopressors, such as phenylephrine or ephedrine, which should be given very cautiously and in much smaller doses than those used in non-preeclamptic patients due to the exaggerated vasoconstrictor response in parturient suffering from pre-eclampsia (Cotoia et al., 2017). Three prospective trials have demonstrated that pre-eclamptic parturient experience less frequent and less sever hypotension and require smaller doses of vasopressors than normotensive controls after initiation of spinal anesthesia (Henkeet al., 2013).

The present work aimed to determine how pre-eclampsia affects maternal hemodynamics during Cesarean section after spinal anesthesia.

\section{PATIENTS AND METHODS}

This prospective randomized and controlled study was carried out on 30 severely preeclamptic females and 30 normotensive females. The study was conducted in the Obstetrics Department of Al-Azhar University Hospitals (AlHussein and Bab-AlShaarya), Cairo, Egypt, from January 2019 till December 2019. After approval from ethical committee, an informed consent was obtained from all patients.

All data of the patients should be confidential with secret code and private file for each patient.

\section{Inclusion criteria:}

- Age: 18 - 40 years

- Severely Pre-eclamptic females, severe pre-eclampsia is defined as hypertension (BP> 159/109), proteinuria (urinary protein excretion of greater than $5 \mathrm{gm}$ per day) and oliguria (urine output $<500 \mathrm{ml}$ in 24 hours) with at least one maternal organ dysfunction.

\section{Exclusion criteria:}

- Patient refusal.

- Age: <18 years.

- Obese patients with BMI > $35 \mathrm{Kg} / \mathrm{m} 2$.

- Preterm delivery.

- Patients with contraindication to spinal anesthesia.

- Patients in active labour.

- Chronic illness e.g. chronic hypertension, renal failure. 
- Congenital anomalies of the fetus.

\section{Preoperative assessment:}

- History (medical and surgical).

- Physical examination.

- Laboratory investigations $(\mathrm{CBC}$, renal function tests, liver function tests. coagulation profile).

\section{Premedication:}

- Slow IV infusion of $50 \mathrm{mg}$ Ranitidine and $10 \mathrm{mg}$ Metoclopramide.

- For seizure prophylaxis, Mgso4 will be given to all severely pre- eclamptic patients, loading dose 4 gm. over 30 minutes followed by $1 \mathrm{gm} /$ hour.

\section{Anesthetic technique:}

The parturient was allocated into one of two equal groups $(\mathrm{n}=30)$, a normotensive group (A) and severe preeclampsia group (B). All was received colloid (500 ml hydroxethyl starch) as a coload via wide bore (18 Gauge) cannula within 5-10 minutes during induction of spinal anesthesia. Standard monitoring with electrocardiography, automated noninvasive arterial pressure (NIAP) measurement, and pulse oximetry will be performed. Systolic arterial pressure (SAP), mean arterial pressure (MAP) and diastolic arterial pressure (DAP) was monitored. Baseline values were recorded in the supine position.

Spinal anesthesia was induced with a total of $10 \mathrm{mg}$ of $0.5 \%$ hyperbaric bupivacaine and $25 \mu \mathrm{g}$ fentanyl (total volume $2.5 \mathrm{ml}$ ) at the $\mathrm{L} 3-4$ interspace in the sitting position then the patient returned immediately to the supine position with left lateral tilt.
Hypotension defined as decrease in systolic blood pressure (SAP) below 30\% of baseline in severe preeclampsia group and below $30 \%$ or developing manifestations of hypotension e.g. nausea and vomiting, and bradycardia defined as decrease in HR below 55 beat / minute.

Ephedrine boluses of 5 or $10 \mathrm{mg}$ were given to correct hypotension and atropine $(0.01 \mathrm{mg} / \mathrm{kg})$ was given to correct bradycardia.

\section{Data collection:}

- Demographic data: (age, weight, height, BMI, Gestational age).

\section{- Hemodynamic data:}

- Heart rate, systolic, diastolic and mean arterial blood pressure pre and post spinal injection (1 minute after spinal injection then every 2.5 minute till delivery of the baby then every 5 minute till the end of surgery).

- Dose of Ephedrine required correcting hypotension.

- Doses of Atropine required correcting bradycardia.

- Oxygen saturation using pulse oximeter reading recorded every 5 minutes.

- Apgar score at 1 and 5 min will be recorded.

- Umbilical artery blood gases.

- Other data collection: headache, vomiting, respiratory distress (Dyspnea or Bradypnea), pulmonary edema and blurring of vision.

\section{Primary outcome:}

To compare incidence of hypotension after spinal anesthesia in severely 
preeclamptic parturient versus normotensive control.

\section{Secondary outcome:}

- To determine ephedrine dose required to correct hypotension.

- To detect APGAR score and umbilical artery blood gases after spinal anesthesia.

- To detect incidence of headache, vomiting, respiratory distress, pulmonary edema and blurring of vision.

\section{Statistical analysis:}

Data management and statistical analysis were performed using the Statistical Package for Social Sciences
(SPSS) version 20.Numerical data were summarized using means and standard deviations or medians and ranges. Data were explored for normally using Kolmogrov-Smirnov test and ShapiroWilk test. Categorical data were summarized as percentages. Comparisons between the 2 groups with respect to normally distributed numeric variables were done using the Independent t-test. Non normally distributed numeric variables were compared by MannWhitney test. For categorical variables, differences were analyzed with (X2)(chi square) test and Fisher's exact test when appropriate. P-values $<0.05$ were considered significant.

\section{RESULTS}

There was no statistically significant difference between two groups regarding their demographic data where $\mathrm{p}$-vale was $>0.05$ (Table 1).

Table (1): Comparison between two groups regarding their Demographic data

\begin{tabular}{|c|c|c|c|c|c|c|c|c|c|}
\hline \multirow{2}{*}{$\begin{array}{l}\text { Gemographic } \\
\text { data }\end{array}$} & \multicolumn{4}{|c|}{$\begin{array}{c}\text { Normotensive group } \\
\mathbf{N = 3 0 )}\end{array}$} & \multicolumn{3}{c|}{$\begin{array}{c}\text { Severe preeclampsia group } \\
(\mathbf{N = 3 0 )}\end{array}$} & T-test \\
\hline & Range & Mean & SD & \multicolumn{2}{|c|}{ Range } & Mean & SD & P-value \\
\hline Age (years) & 21 & 37 & 28.7 & 4.34 & 20 & 39 & 28.93 & 4.59 & 0.842 \\
\hline BMI & 25.4 & 33.3 & 29.73 & 1.89 & 24.7 & 33.7 & 30.03 & 2.36 & 0.588 \\
\hline GA (wks) & 36 & 39 & 37.53 & 0.86 & 36 & 39 & 37.3 & 0.88 & 0.310 \\
\hline
\end{tabular}

There was a highly statistically significant difference between two groups regarding DBP data where p-vale was $<0.001$ (Table 2). 
Table (2): Comparison between two groups regarding DBP ( $\mathrm{mmHg}$ )

\begin{tabular}{|c|c|c|c|c|c|c|c|}
\hline \multirow{2}{*}{ DBP (mmHg) } & \multicolumn{2}{|c|}{$\begin{array}{c}\text { Normotensive } \\
\text { group (N=30) }\end{array}$} & \multicolumn{2}{c|}{$\begin{array}{c}\text { Severe preeclampsia } \\
\text { group (N=30) }\end{array}$} & T-test \\
\cline { 2 - 8 } & Mean & \pm & SD & Mean & \pm & SD & P-value \\
\hline Baseline & 63.6 & \pm & 5.75 & 98.62 & \pm & 8.78 & $<0.001$ \\
\hline 1 min. after spinal & 50.7 & \pm & 8.87 & 85.35 & \pm & 5.05 & $<0.001$ \\
\hline 2.5 min. after spinal & 52.5 & \pm & 5.47 & 90.47 & \pm & 9.21 & $<0.001$ \\
\hline 5 min. after spinal & 51.4 & \pm & 9.66 & 89.21 & \pm & 9.57 & $<0.001$ \\
\hline 7.5 min. after spinal & 53.8 & \pm & 6.92 & 83.81 & \pm & 6.91 & $<0.001$ \\
\hline 10 min. after spinal & 54.34 & \pm & 9.76 & 81.34 & \pm & 5.65 & $<0.001$ \\
\hline 12.5 min. after spinal & 54.6 & \pm & 7.33 & 80.38 & \pm & 6.46 & $<0.001$ \\
\hline 15 min. after spinal & 55.89 & \pm & 9.21 & 80.62 & \pm & 7.05 & $<0.001$ \\
\hline 17.5 min. after spinal & 56.43 & \pm & 6.47 & 81.4 & \pm & 7.55 & $<0.001$ \\
\hline At delivery & 58.12 & \pm & 7.18 & 82.5 & \pm & 5.11 & $<0.001$ \\
\hline 5 min. after delivery & 58.22 & \pm & 5.91 & 81.48 & \pm & 8.85 & $<0.001$ \\
\hline 10 min. after delivery & 59.69 & \pm & 5.11 & 80.62 & \pm & 8.85 & $<0.001$ \\
\hline 15 min. after delivery & 61.47 & \pm & 5.82 & 78.6 & \pm & 7.44 & $<0.001$ \\
\hline
\end{tabular}

There was a highly statistically significant difference between two groups regarding SBP data where p-vale was $<0.001$ (Table 3).

Table (3): Comparison between two groups regarding SBP (mmHg)

\begin{tabular}{|c|c|c|c|c|c|c|c|}
\hline \multirow{2}{*}{ SBP(mmHg) } & \multicolumn{3}{|c|}{$\begin{array}{c}\text { Normotensive } \\
\text { group (N=30) }\end{array}$} & \multicolumn{3}{c|}{$\begin{array}{c}\text { Severe preeclampsia } \\
\text { group (N=30) }\end{array}$} & T-test \\
\cline { 2 - 8 } & Mean & \pm & SD & Mean & \pm & SD & P-value \\
\hline Baseline & 128.7 & \pm & 5.54 & 169.6 & \pm & 5.56 & $<0.001$ \\
\hline 1 min. after spinal & 115.6 & \pm & 6.21 & 155.52 & \pm & 5.11 & $<0.001$ \\
\hline 2.5 min. after spinal & 120.3 & \pm & 8.41 & 160.74 & \pm & 7.67 & $<0.001$ \\
\hline 5 min. after spinal & 118.45 & \pm & 9.91 & 157.3 & \pm & 9.94 & $<0.001$ \\
\hline 7.5 min. after spinal & 119.7 & \pm & 7.20 & 155.8 & \pm & 5.96 & $<0.001$ \\
\hline 10 min. after spinal & 121.12 & \pm & 7.44 & 156.12 & \pm & 8.87 & $<0.001$ \\
\hline 12.5 min. after spinal & 115.62 & \pm & 6.26 & 154.6 & \pm & 8.68 & $<0.001$ \\
\hline 15 min. after spinal & 112.9 & \pm & 5.89 & 151.9 & \pm & 6.86 & $<0.001$ \\
\hline 17.5 min. after spinal & 111.15 & \pm & 7.50 & 152.12 & \pm & 9.53 & $<0.001$ \\
\hline At delivery & 109.8 & \pm & 6.45 & 153.3 & \pm & 5.05 & $<0.001$ \\
\hline 5 min. after delivery & 108.1 & \pm & 10.5 & 154.7 & \pm & 9.69 & $<0.001$ \\
\hline 10 min. after delivery & 108.6 & \pm & 7.91 & 152.65 & \pm & 5.63 & $<0.001$ \\
\hline 15 min. after delivery & 110.5 & \pm & 8.62 & 150.1 & \pm & 9.87 & $<0.001$ \\
\hline
\end{tabular}

A highly statistically significant difference between two groups regarding
MBP data where $\mathrm{p}$-vale was $<0.001$ (Table 4). 
Table (4): Comparison between two groups regarding MBP (mmHg)

\begin{tabular}{|c|c|c|c|c|c|c|c|}
\hline \multirow{2}{*}{ MBP(mmHg) } & \multicolumn{3}{|c|}{$\begin{array}{c}\text { Gormotensive } \\
\text { group }(\mathbf{N = 3 0})\end{array}$} & \multicolumn{2}{c|}{$\begin{array}{c}\text { Severe preeclampsia } \\
\text { group (N=30) }\end{array}$} & T-test \\
\cline { 2 - 8 } & Mean & \pm & SD & Mean & \pm & SD & P-value \\
\hline Baseline & 98.6 & \pm & 6.08 & 133.6 & \pm & 6.94 & $<0.001$ \\
\hline 1 min. after spinal & 82.7 & \pm & 7.41 & 125.3 & \pm & 7.16 & $<0.001$ \\
\hline 2.5 min. after spinal & 85.2 & \pm & 8.55 & 124.4 & \pm & 5.92 & $<0.001$ \\
\hline 5 min. after spinal & 83.4 & \pm & 7.70 & 121.15 & \pm & 8.68 & $<0.001$ \\
\hline 7.5 min. after spinal & 86.32 & \pm & 8.04 & 119.62 & \pm & 9.32 & $<0.001$ \\
\hline 10 min. after spinal & 86.7 & \pm & 9.46 & 118.7 & \pm & 5.25 & $<0.001$ \\
\hline 12.5 min. after spinal & 84 & \pm & 8.53 & 117.5 & \pm & 5.14 & $<0.001$ \\
\hline 15 min. after spinal & 83.84 & \pm & 5.61 & 116.09 & \pm & 5.14 & $<0.001$ \\
\hline 17.5 min. after spinal & 83.62 & \pm & 9.96 & 117.4 & \pm & 5.87 & $<0.001$ \\
\hline At delivery & 82.7 & \pm & 7.92 & 115.12 & \pm & 9.85 & $<0.001$ \\
\hline 5 min. after delivery & 84.26 & \pm & 8.81 & 116.7 & \pm & 8.46 & $<0.001$ \\
\hline 10 min. after delivery & 85.31 & \pm & 7.94 & 117.4 & \pm & 8.94 & $<0.001$ \\
\hline 15 min. after delivery & 86.05 & \pm & 7.60 & 114.6 & \pm & 6.53 & $<0.001$ \\
\hline
\end{tabular}

A statistically significant difference between two groups regarding

Hypotension data where p-vale was $<0.05$ (Table 5).

Table (5): Comparison between two groups regarding Hypotension

\begin{tabular}{|c|c|c|c|c|c|c|c|}
\hline \multirow{2}{*}{\multicolumn{2}{|c|}{ Hypotension Groups }} & \multicolumn{2}{|c|}{$\begin{array}{l}\text { Normotensive } \\
\text { group }(\mathbf{N}=30)\end{array}$} & \multicolumn{2}{|c|}{$\begin{array}{l}\text { Severe preeclampsia } \\
\text { group }(\mathbf{N}=30)\end{array}$} & \multicolumn{2}{|c|}{ Total } \\
\hline & & $\mathrm{N}$ & $\%$ & $\mathrm{~N}$ & $\%$ & $\mathrm{~N}$ & $\%$ \\
\hline \multicolumn{2}{|c|}{ Yes } & 20 & 66.7 & 9 & 30.0 & 29 & 48.3 \\
\hline \multicolumn{2}{|c|}{$\mathrm{No}$} & 10 & 33.3 & 21 & 70.0 & 31 & 51.7 \\
\hline \multicolumn{2}{|c|}{ Total } & 30 & 100.0 & 30 & 100.0 & 60 & 100.0 \\
\hline \multirow{2}{*}{ Chi-square } & $\mathrm{X}^{2}$ & \multicolumn{6}{|c|}{8.076} \\
\hline & P-value & \multicolumn{6}{|c|}{0.004} \\
\hline
\end{tabular}

There was highly statistically significant difference between two groups regarding total dose of ephedrine data where p-vale was $<0.001$ while was no statistically significant difference in total dose of atropine where p-vale was $>0.05$ (Table 6).

Table (6): Comparison between two groups regarding Total dose of Ephedrine and Atropine

\begin{tabular}{|c|c|c|c|c|c|c|c|c|c|c|c|}
\hline \multirow{2}{*}{ Groups } & \multicolumn{9}{|c|}{$\begin{array}{c}\text { Normotensive group } \\
\mathbf{( N = 3 0 )}\end{array}$} & \multicolumn{7}{c|}{$\begin{array}{c}\text { Severe preeclampsia group } \\
\mathbf{N = 3 0 )}\end{array}$} & \multicolumn{2}{|c|}{} \\
\cline { 2 - 12 } & Range & Mean & SD & Median & Range & Mean & SD & Median & P-value \\
\hline $\begin{array}{c}\text { Total dose } \\
\text { of Ephedrine }\end{array}$ & 0 & 30 & 9.83 & 9.30 & 9.50 & 0 & 18 & 3 & 5.15 & 0.00 & $<0.001 * *$ \\
\hline $\begin{array}{c}\text { Total dose } \\
\text { of Atropine }\end{array}$ & 0 & 1 & 0.07 & 0.22 & 0.00 & 0 & 0.50 & 0.02 & 0.09 & 0.00 & 0.254 \\
\hline
\end{tabular}

A highly statistically significant difference between two groups regarding
Headache and Blurring of vision data where p-vale was $<0.001$ (Table 7). 
Table (7): Comparison between two groups regarding their Complications

\begin{tabular}{|c|c|c|c|c|c|c|}
\hline \multirow{2}{*}{ Groups } & $\begin{array}{c}\text { Normotensive } \\
\text { group }(\mathbf{N = 3 0 )}\end{array}$ & $\begin{array}{c}\text { Severe preeclampsia } \\
\text { group (N=30) }\end{array}$ & \multicolumn{2}{c|}{ Chi-square } \\
\cline { 2 - 7 } & $\mathrm{N}$ & $\%$ & $\mathrm{~N}$ & $\%$ & $\mathrm{X}^{2}$ & P-value \\
\hline Complications & 0 & 0.0 & 12 & 40.0 & 15.000 & $<0.001^{* *}$ \\
\hline Headache & 0 & 0.0 & 6 & 20.0 & 6.667 & $0.010^{*}$ \\
\hline Blurring of vision & 9 & 30.0 & 7 & 23.3 & 0.341 & 0.559 \\
\hline Vomiting & 0 & 0.0 & 1 & 3.3 & 1.017 & 0.313 \\
\hline
\end{tabular}

There was no statistically significant difference between two groups regarding
Fetal acidosis where p-vale was $>0.05$ (Table 8).

Table (8): Comparison between two groups regarding their Fetal Acidosis

\begin{tabular}{|c|c|c|c|c|c|c|c|}
\hline \multirow{2}{*}{\multicolumn{2}{|c|}{ Fetal acidosis Groups }} & \multicolumn{2}{|c|}{$\begin{array}{l}\text { Normotensive } \\
\text { group }(\mathbf{N}=30)\end{array}$} & \multicolumn{2}{|c|}{$\begin{array}{l}\text { Severe preeclampsia } \\
\text { group }(\mathbf{N}=\mathbf{3 0})\end{array}$} & \multicolumn{2}{|c|}{ Total } \\
\hline & & $\mathrm{N}$ & $\%$ & $\mathrm{~N}$ & $\%$ & $\mathrm{~N}$ & $\%$ \\
\hline \multicolumn{2}{|c|}{ Normal } & 26 & 86.7 & 24 & 80.0 & 50 & 83.3 \\
\hline \multicolumn{2}{|c|}{ Acidosis } & 4 & 13.3 & 6 & 20.0 & 10 & 16.7 \\
\hline \multicolumn{2}{|c|}{ Total } & 30 & 100 & 30 & 100 & 60 & 100 \\
\hline \multirow{2}{*}{ Chi-square } & $\mathrm{X}^{2}$ & \multicolumn{6}{|c|}{0.480} \\
\hline & P-value & \multicolumn{6}{|c|}{0.488} \\
\hline
\end{tabular}

\section{DISCUSSION}

The current study was conducted on 30 severely preeclamptic females and 30 normotensive females. It was aimed to determine how pre-eclampsia affects maternal hemodynamics during Cesarean section after spinal anesthesia.

Regarding demographic data showed no statistically significant differences between the two groups.

We found that incidence of hypotension after spinal anesthesia in group A (normotensive) was $66.7 \%$ and in group B (preeclampsia) was $30 \%$.

These results went in line with that of Goel et al. (2018) who found that the incidence of hypotension, as defined by a $30 \%$ decrease in mean BP, is less in patients with severe preeclampsia undergoing spinal anesthesia for cesarean delivery, as compared with healthy parturient. In addition, the magnitude of the decrease in mean BP is smaller in severely preeclamptic patients. Also our results were similar to the follow up study of Henkeet al. (2013) as they demonstrated that the incidence of clinically relevant hypotension (decrease in MAP to $<70 \%$ ) leading to ephedrine treatment was lower in the severely preeclamptic group compared with the preterm group ( $24.4 \%$ versus $40.8 \%$ ).

Dyer et al. (2010) showed results against ours. They concluded that spinal anesthesia in severe preeclampsia was associated with significant decrease in MAP and systemic vascular resistance from the time of adoption of the supine position until the end of surgery, however there is insignificant changes in CO. This study has relatively small sample size (n $=15$ ) also CVP was not measured in this study. 
The mean ephedrine requirement of the normotensive group $(27.9+/-11.6 \mathrm{mg})$ was significantly greater $(\mathrm{P}<0.01)$ than that of the preeclamptic group $(16.4+/-15.0$ mg)and it was similar to the study of Cartyet al. (2010). The preeclamptic patients had a less frequent incidence of clinically significant hypotension, which was less severe and required less ephedrine (Chaudharyet al., 2011).

Regarding complications including headache, blurring of vision, vomiting and respiratory distress (bradypnea or distress), we found that incidence of headache in group B was $40 \%$ and incidence of blurring of vision was $20 \%$. While incidence of vomiting and respiratory distress were comparable.

Regarding neonates, APGAR score showed no statistically significant differences between the two groups at 1 $\min \& 5$ min.

These results went in line with the results of Henke et al. (2013), they reported comparable APGAR score in both groups.

\section{CONCLUSION}

Incidences of hypotension after spinal anesthesia are less frequent and less severe in preeclampsia patients and require lower dose of ephedrine than normotensive population. There was increased incidence of headache and blurring of vision among severe preeclampsia parturient. No difference in neonatal outcome between normotensive and severe preeclampsia population following spinal anesthesia.

\section{REFERENCES}

1. Banerjee, A., Stocche, R. M., Angle, P. and Halpern, S. H. (2010): Preload or coload for spinal anesthesia for elective Cesarean delivery: a meta-analysis. Canadian Journal of Anesthesia/Journal canadiend' anesthésie, 57(1): 24-31.

2. Carty, D. M., Delles, C., and Dominiczak, A. F. (2010): Preeclampsia and future maternal health. Journal of hypertension, 28(7):1349-1355.

3. Chaudhary, S., and Salhotra, R. (2011): Subarachnoid block for caesarean section in severe preeclampsia. Journal of Anesthesiology, Clinical Pharmacology, 27(2):169-172.

4. Cotoia, A., Mirabella, L., Raimondo, P. and Cinnella, G. (2017): Complications of regional anesthesia. In Management and Therapy of Late Pregnancy Complications Springer, Cham.p. 265-293.

5. Dyer, R. A., Reed, A. R., van Dyk, D., Arcache, M. J., Hodges, O., Lombard, C. J. and James, M. F.(2010): Hemodynamic effects of ephedrine, phenylephrine, and the co administration of phenylephrine with oxytocin during spinal anesthesia for elective cesarean delivery. Obstetric Anesthesia Digest, 30(3): 160-163.

6. Goel, L., Cordeiro, R., and Goel, M. (2018): Intraoperative requirement of Phenylephrine for spinal anesthesia, with comparison of hemodynamic parameters between severe pre-eclamptic and normotensive parturients for elective caesarean section: A prospective study. Indian Journal of Clinical Anaesthesia, 5(1):125-128.

7. Henke, V. G., Bateman, B. T. and Leffert, L. R. (2013): Spinal anesthesia in severe preeclampsia. Anesthesia\& Analgesia, 117(3):686-693. 
8. Liguori, G. A. (2012): Hemodynamic complications. Complications in Regional Anesthesia and Pain Medicine, 59 (6): 847-847.

9. Tayde, P. V., Ansari ${ }^{1}$, A., Loutf $y^{1}$, I. and Sharma, H. (2018): Management of Perioperative Pulmonary Edema in a Case of Preeclampsia for Cesarean Section. Journal of Anaesthesia and Critical Care Case Reports, 4(2): 15-17.

10.Tranquilli, A. L., Brown, M. A., Zeeman, G. G. Dekker, G., and Sibai, B. M. (2013): The definition of severe and early-onset preeclampsia. Statements from the International Society for the Study of Hypertension in Pregnancy (ISSHP).
Pregnancy Hypertension: An International Journal of Women's Cardiovascular Health, 3(1): 44-47.

11.Turner, J. A. (2010): Diagnosis and management of pre-eclampsia: an update. International Journal of Women's Health, 2: 327-328. 


\section{التغيرات الديناميكية الاموية المصاحبة للتخدير التصفي خلال

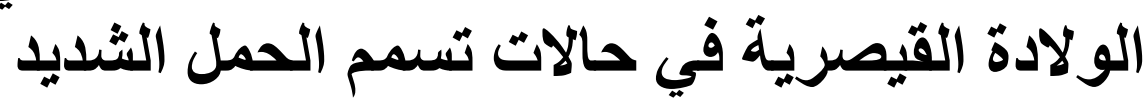

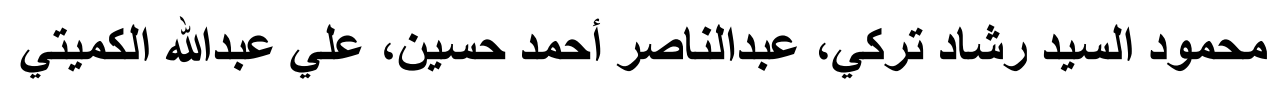
قسم التخدير والرعاية المركزة، كلية طب الأزهر

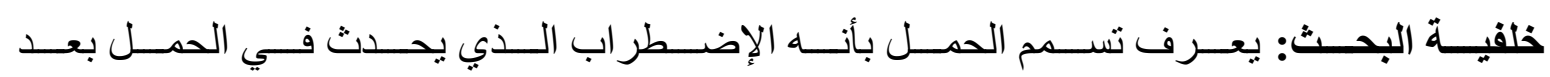

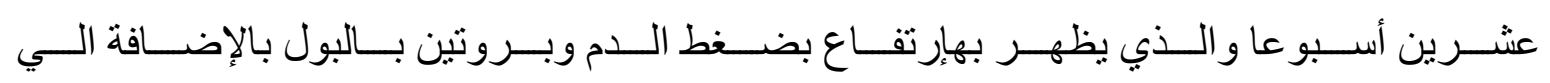
خلل بأجهزة الأم (واحدة على الأقل).

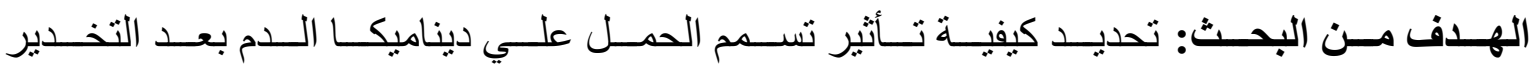
النصفي أثناء الو لادة القيصرية.

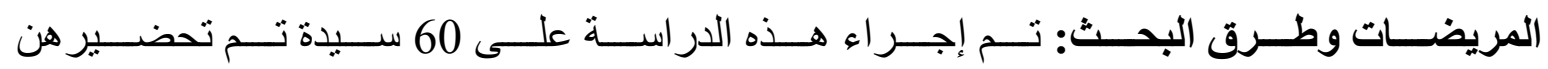

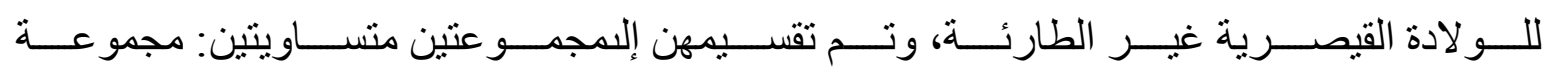

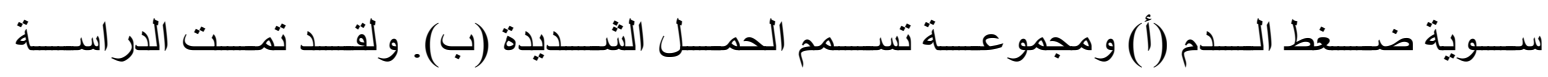

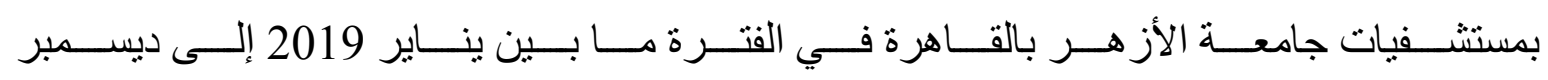

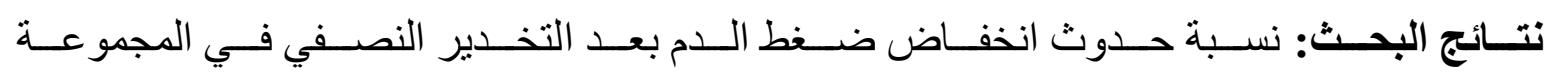

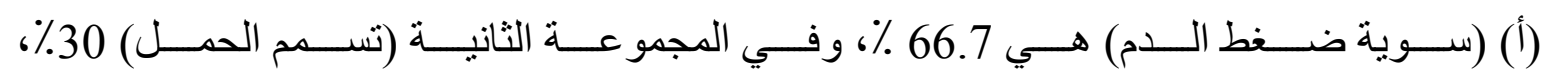
النتائج الوليدية (نتيجة الابجر و غاز ات الدم من الثريان السري) قابلة للمقارنة.

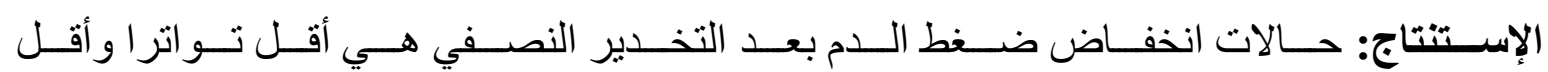

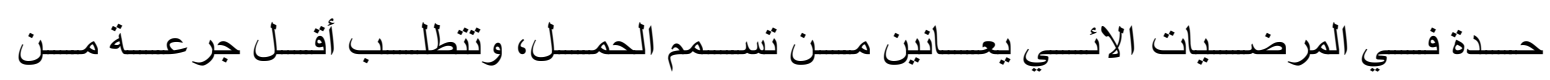

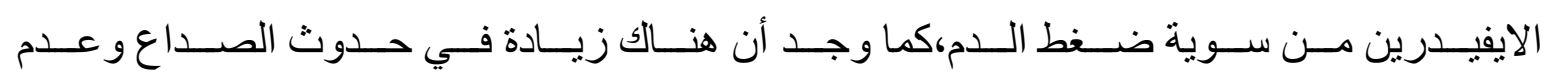

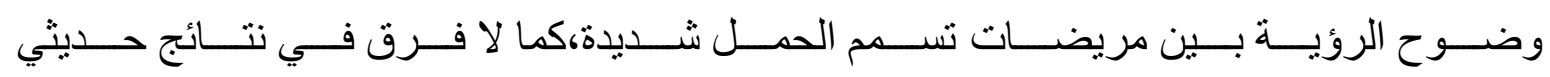
الو لادة بين مريضات تسمم الحمل الثديد وسوية الضغط بعد التخدير النصفي. 